\title{
A CONSISTENT MARKOV PARTITION PROCESS GENERATED FROM THE PAINTBOX PROCESS
}

\author{
HARRY CRANE, ${ }^{*}$ University of Chicago
}

\begin{abstract}
We study a family of Markov processes on $\mathcal{P}^{(k)}$, the space of partitions of the natural numbers with at most $k$ blocks. The process can be constructed from a Poisson point process on $\mathbb{R}^{+} \times \prod_{i=1}^{k} \mathcal{P}^{(k)}$ with intensity $\mathrm{d} t \otimes \varrho_{v}^{(k)}$, where $\varrho_{v}$ is the distribution of the paintbox based on the probability measure $v$ on $\mathcal{P}_{\mathrm{m}}$, the set of ranked-mass partitions of 1 , and $\varrho_{v}^{(k)}$ is the product measure on $\prod_{i=1}^{k} \mathcal{P}^{(k)}$. We show that these processes possess a unique stationary measure, and we discuss a particular set of reversible processes for which transition probabilities can be written down explicitly.
\end{abstract}

Keywords: Paintbox process; Ewens' partition; Poisson-Dirichlet distribution; partition process

2010 Mathematics Subject Classification: Primary 60J25

Secondary 60G09

\section{Introduction}

Markov processes on the space of partitions appear in a variety of situations in the scientific literature, such as, but not limited to, physical chemistry, astronomy, and population genetics. See [1] for a relatively recent overview of this literature. Well-behaved mathematically tractable models of random partitions are of interest to probabilists as well as statisticians and scientists; see [10], [12], [13], and [15]. Ewens [10] first introduced the Ewens' sampling formula in the context of theoretical population biology. Kingman's [12] coalescent model was introduced as a model for population genetics, still its most natural setting. However, since the seminal works of Ewens and Kingman, random partitions have appeared in areas ranging from classification models, as in [7] and [15], to probability theory (see [3] and [17]). McCullagh [13] described how Ewens' model can be used in the classical problem of estimating the number of unseen species, introduced by Fisher [11] and later studied by many, including Efron and Thisted [9].

Berestycki [2] studied a family of partition processes, called exchangeable fragmentationcoalescence (EFC) processes, whose paths are generated by a combination of independent coalescent and fragmentation processes. The mathematical tractability of coalescent and fragmentation processes has led to the development of many results for EFC processes and has led to interest in more complex models. For a sample of these results and relevant references, see [3], [16], and [17]. The study of processes, such as the EFC process, which admit a more general study of partition-valued processes is of interest from a theoretical as well as applied perspective. In this paper, we study a family of processes which is similar in spirit to the EFC process, but whose sample paths are quite different.

Received 6 January 2011; revision received 28 February 2011.

* Postal address: Department of Statistics, University of Chicago, Eckhart Hall Room 108, 5734 South University Avenue, Chicago, IL 60637, USA. Email address: crane@uchicago.edu 


\section{Preliminaries}

Throughout this paper, $\mathcal{P}$ denotes the space of set partitions of the natural numbers $\mathbb{N}$. We regard an element $B$ of $\mathcal{P}$ as a collection of disjoint nonempty subsets, called blocks, written $B=\left\{B_{1}, B_{2}, \ldots\right\}$, such that $\bigcup_{i} B_{i}=\mathbb{N}$. The blocks are unordered, but, where necessary, they are listed in the order of their least element. We write $B=\left(B_{1}, B_{2}, \ldots\right)$ whenever we wish to emphasize that blocks are listed in a particular order. For $B \in \mathcal{P}$ and $b \in B$, \#B is the number of blocks of $B$ and $\# b$ is the number of elements of $b$. For any $A \subset \mathbb{N}$, let $B_{\mid A}$ denote the restriction of $B$ to $A$. Wherever necessary, $\mathcal{P}^{(k)}$ denotes the space of partitions of $\mathbb{N}$ with at most $k$ blocks, i.e. $\mathcal{P}^{(k)}:=\{B \in \mathcal{P}: \# B \leq k\}$. For fixed $n \in \mathbb{N}, \mathcal{P}_{[n]}$ and $\mathcal{P}_{[n]}^{(k)}$ are the restrictions to $[n]:=\{1, \ldots, n\}$ of $\mathcal{P}$ and $\mathcal{P}^{(k)}$, respectively.

It is sometimes convenient to regard a partition $B$ as either an equivalence relation defined by $B(i, j)=1 \Leftrightarrow i \sim_{B} j$ or an $n \times n$ symmetric Boolean matrix whose $(i, j)$ th entry is $B(i, j)$. These three representations are equivalent and we use the same notation to refer to any one of them.

For each $\pi, \pi^{\prime} \in \mathcal{P}$, we define the metric $d: \mathcal{P} \times \mathcal{P} \rightarrow \mathbb{R}$ such that

$$
d\left(\pi, \pi^{\prime}\right)=\frac{1}{\max \left\{n \in \mathbb{N}: \pi_{\mid[n]}=\pi_{\mid[n]}^{\prime}\right\}} .
$$

The space $(\mathcal{P}, d)$ is compact [5].

In addition, we define the projection $D_{m, n}: \mathcal{P}_{[n]} \rightarrow \mathcal{P}_{[m]}$ for each $n \geq m \geq 1$ by $D_{m, n} B_{[n]}=$ $B_{[n] \mid[m]}$. In the matrix representation, $D_{m, n} B$ is the leading $m \times m$ submatrix of $B$. We seek processes $B:=\left(B_{t}, t \geq 0\right)$ on $\mathcal{P}$ such that, for each $n \in \mathbb{N}$, the restriction of $B$ to $[n], B_{\mid[n]}$, is finitely exchangeable and consistent. That is,

- $\sigma\left(B_{\mid[n]}\right) \sim B_{\mid[n]}$ for each $\sigma \in \S_{n}$, the symmetric group acting on [n]; and

- $B_{[n] \mid[m]} \sim B_{\mid[m]}$ for each $m<n$.

It is more convenient to work with $\mathcal{P}$ as the state space of our process than the space $\mathcal{P}_{\mathrm{m}}=$ $\left\{\left(s_{1}, s_{2}, \ldots\right): s_{1} \geq s_{2} \geq \cdots \geq 0, \sum_{i} s_{i} \leq 1\right\}$ of ranked-mass partitions of $x \in[0,1]$. In accordance with the notation for set partitions, let $\mathcal{P}_{\mathrm{m}}^{(k)}:=\left\{s \in \mathcal{P}_{\mathrm{m}}: s_{j}=0\right.$ for all $j>k$, $\left.\sum_{i=1}^{k} s_{i}=1\right\}$ denote the ranked $k$-simplex. There is an intimate relationship between exchangeable processes on $\mathcal{P}$ and processes on $\mathcal{P}_{\mathrm{m}}$ through the paintbox process.

For $s \in \mathcal{P}_{\mathrm{m}}$, let $X:=\left(X_{1}, X_{2}, \ldots\right)$ be independent random variables with distribution

$$
\mathrm{P}_{s}\left(X_{i}=j\right)= \begin{cases}s_{j}, & j \geq 1, \\ 1-\sum_{i=1}^{\infty} s_{i}, & j=-i \\ 0, & \text { otherwise }\end{cases}
$$

The partition $\Pi(X)$ generated by $s$ through $X$ satisfies $i \sim_{\Pi(X)} j$ if and only if $X_{i}=X_{j}$. The distribution of $\Pi(X)$ is written $\varrho_{s}$ and $\Pi(X)$ is called the paintbox based on $s$. For a measure $v$ on $\mathcal{P}_{\mathrm{m}}$, the paintbox based on $v$ is the $\nu$-mixture of paintboxes, written $\varrho_{v}(\cdot):=\int_{\mathcal{P}_{\mathrm{m}}} \varrho_{s}(\cdot) \nu(\mathrm{d} s)$. Any partition obtained in this way is an exchangeable random partition of $\mathbb{N}$ and every infinitely exchangeable partition admits a representation as the paintbox generated by some $v$. See [5] and [17] for more details on the paintbox process. 
We are particularly interested in exchangeable Markovian transition probabilities $\left(p_{n}\right)$, where, for every $n, p_{n}$ is a transition probability on $\mathcal{P}_{[n]}$ which satisfies

$$
p_{n}\left(B, B^{\prime}\right)=\sum_{B^{\prime \prime} \in D_{n, n+1}^{-1}\left(B^{\prime}\right)} p_{n+1}\left(B^{*}, B^{\prime \prime}\right)
$$

for each $B, B^{\prime} \in \mathcal{P}_{[n]}$ and $B^{*} \in D_{n, n+1}^{-1}(B)$. Burke and Rosenblatt [8] showed that (2.1) is necessary and sufficient for $\left(p_{n}\right)$ to be consistent under selection from $\mathbb{N}$.

Likewise, for a continuous-time Markov process, $\left(B_{n}(t), t \geq 0\right)_{n \in \mathbb{N}}$, where $B_{n}(t)$ is a process on $\mathcal{P}_{[n]}$ with infinitesimal generator $Q_{n}$, it is sufficient that the entries of $Q_{n}$ satisfy (2.1) for there to be a Markov process on $\mathcal{P}$ with those finite-dimensional transition rates.

\section{The $\varrho_{v}$-Markov chain on $\mathcal{P}^{(k)}$}

Let $n, k \in \mathbb{N}$, and let $v$ be a probability measure on the ranked $k$-simplex $\mathcal{P}_{\mathrm{m}}^{(k)}$, so that the paintbox based on $v$ is obtained by a conditionally independent and identically distributed (i.i.d.) sample from $v$, i.e. given $s \sim v, X_{1}, X_{2}, \ldots$ are i.i.d. with $\mathrm{P}_{s}\left(X_{i}=j\right)=s_{j}$ for each $j=1, \ldots, k$. For convenience, we write $B \in \mathcal{P}^{(k)}$ as an ordered list $\left(B_{1}, \ldots, B_{k}\right)$, where $B_{i}$ corresponds to the $i$ th block of $B$ in order of appearance for $i \leq \# B$ and $B_{i}=\varnothing$ for $i=\# B+1, \ldots, k$.

Consider the following Markov transition operation $B \mapsto B^{\prime}$ on $\mathcal{P}^{(k)}$. Let $B=\left(B_{1}, \ldots\right.$, $\left.B_{k}\right) \in \mathcal{P}^{(k)}$ and, independently of $B$, generate $C_{1}, C_{2}, \ldots$ which are i.i.d. according to $\varrho_{v}$. For each $i$, we write $C_{i}:=\left(C_{i 1}, \ldots, C_{i k}\right) \in \mathcal{P}^{(k)}$. Independently of $B, C_{1}, C_{2}, \ldots$, generate $\sigma_{1}, \sigma_{2}, \ldots$, which are independent uniform random permutations of $[k]$. Given $\sigma:=$ $\left(\sigma_{1}, \sigma_{2}, \ldots, \sigma_{k}\right)$, we arrange $B, C_{1}, \ldots, C_{k}$ in matrix form as follows:

$$
\begin{gathered}
C_{.1} \\
B_{1} \\
B_{2} \\
\vdots \\
B_{k}
\end{gathered}\left(\begin{array}{cccc}
C_{1, \sigma_{1}(1)} \cap B_{1} & C_{1, \sigma_{1}(2)} \cap B_{1} & \cdots & C_{1, \sigma_{1}(k)} \cap B_{1} \\
C_{2, \sigma_{2}(1)} \cap B_{2} & C_{2, \sigma_{2}(2)} \cap B_{2} & \cdots & C_{2, \sigma_{2}(k)} \cap B_{2} \\
\vdots & \vdots & \ddots & \vdots \\
C_{k, \sigma_{k}(1)} \cap B_{k} & C_{k, \sigma_{k}(2)} \cap B_{k} & \cdots & C_{k, \sigma_{k}(k)} \cap B_{k}
\end{array}\right)=: B \cap C^{\sigma} .
$$

Here $B \cap C^{\sigma}$ is a matrix with row totals corresponding to the blocks of $B$ and column totals $C_{. j}=\bigcup_{i=1}^{k}\left(C_{i, \sigma_{i}(j)} \cap B_{i}\right)$. Finally, $B^{\prime}$ is obtained as the collection of nonempty blocks of $\left(C_{.1}, \ldots, C_{. k}\right)$. The nonempty entries of $B \cap C^{\sigma}$ form a partition in $\mathcal{P}^{\left(k^{2}\right)}$ which corresponds to the greatest lower bound $B \wedge B^{\prime}$.

Proposition 3.1. The above description gives rise to finite-dimensional transition probabilities on $\mathcal{P}_{[n]}^{(k)}$ :

$$
p_{n}\left(B, B^{\prime} ; v\right)=\frac{k !}{\left(k-\# B^{\prime}\right) !} \prod_{b \in B} \frac{\left(k-\# B_{\mid b}^{\prime}\right) !}{k !} \varrho_{\nu}\left(B_{\mid b}^{\prime}\right) .
$$

Proof. Let $A \in \mathcal{P}^{(k)}$. Fix $n, k \in \mathbb{N}$, and set $B:=A_{\mid[n]} \in \mathcal{P}_{[n]}^{(k)}$. Let $C_{1}, \ldots, C_{k}$ be i.i.d. $\varrho_{\nu}$-distributed partitions, and let $\sigma:=\left(\sigma_{1}, \ldots, \sigma_{k}\right)$ be i.i.d. uniform random permutations of $[k]$ as described above. Let $B^{\prime}$ be the set partition obtained from the column totals of the matrix $B \cap C^{\sigma}$ in the above construction.

From the matrix construction, it is clear that, for each $i=1, \ldots, k$, the restriction $B_{\mid B_{i}}^{\prime}$ is equal to the set partition in $\mathcal{P}_{[n]}^{(k)}$ associated with $C_{i}\left[B_{i}\right]:=\left(C_{i 1} \cap B_{i}, \ldots, C_{i k} \cap B_{i}\right)$. Conversely, 
the transition $B \mapsto B^{\prime}$ occurs only if the collection $\left(C_{1}, \ldots, C_{k}\right)$ is such that, for each $B_{i} \in B$, $C_{i}\left[B_{i}\right]=B_{\mid B_{i}}^{\prime}$. By consistency of the paintbox process, for each $i=1, \ldots, k, C_{i}\left[B_{i}\right]$ has probability

$$
\varrho_{\nu}\left(C_{i}\left[B_{i}\right]\right)=\varrho_{v}\left(B_{\mid B_{i}}^{\prime}\right) .
$$

Independence of the $C_{i}$ implies that the probability of $B \wedge B^{\prime}$ given $B$ is

$$
\prod_{b \in B} \varrho_{\nu}\left(B_{\mid b}^{\prime}\right) .
$$

Finally, each uniform permutation $\sigma_{i}$ has probability $1 / k$ ! and there are $\left(k ! /\left(k-\# B^{\prime}\right) !\right) \times$ $\prod_{b \in B}\left(k-\# B_{\mid b}^{\prime}\right)$ ! collections $\sigma_{1}, \ldots, \sigma_{\# B}$ such that the column totals of $B \cap C^{\sigma}$ correspond to the blocks of $B^{\prime}$. This completes the proof.

For fixed $n$, (3.1) depends only on $B$ and $B^{\prime}$ through $\varrho_{v}$ and the number of blocks of $B$ and $B^{\prime}$, and is, therefore, finitely exchangeable. We appeal to (2.1) to establish consistency.

Proposition 3.2. For any measure $v$ on $\mathcal{P}_{\mathrm{m}}^{(k)}$, let $\left(p_{n}(\cdot, \cdot ; v)\right)_{n \geq 1}$ be the collection of transition probabilities on $\mathcal{P}_{[n]}^{(k)}$ defined in (3.1). Then $\left(p_{n}\right)$ is a consistent family of transition probabilities.

Proof. Fix $n, k \in \mathbb{N}$, and let $B, B^{\prime} \in \mathcal{P}_{[n]}^{(k)}$. To establish consistency, it is enough to verify condition (2.1) from Theorem 1 of [8], i.e. for each $v$ and $B^{*} \in D_{n, n+1}^{-1}(B)$,

$$
p_{n+1}\left(B^{*}, D_{n, n+1}^{-1}\left(B^{\prime}\right) ; v\right)=p_{n}\left(B, B^{\prime} ; v\right) \text {. }
$$

We assume without loss of generality that $B^{*} \in D^{-1}(B)$ is obtained from $B$ by the operation $n+1 \mapsto B_{1} \in B$ and we write $B_{1}^{*}:=B_{1} \cup\{n+1\}$. Likewise, for $B^{\prime \prime} \in D_{n, n+1}^{-1}\left(B^{\prime}\right)$ obtained by $n+1 \mapsto B_{i}^{\prime} \in B^{\prime} \cup\{\varnothing\}$, write $B_{i}^{\prime *}:=B_{i}^{\prime} \cup\{n+1\}$. So either $n+1 \in B_{i}^{\prime *}$ for some $i=1, \ldots, \# B^{\prime}$ or $n+1$ is inserted in $B^{\prime}$ as a singleton.

The change to $B \cap C^{\sigma}$ that results from inserting $n+1$ into $B_{1} \in B$ and $B_{i}^{\prime} \in B^{\prime}$ is summarized by the following matrix (note that $B_{j}^{\prime}=\varnothing$ for $j>\# B^{\prime}$ ):

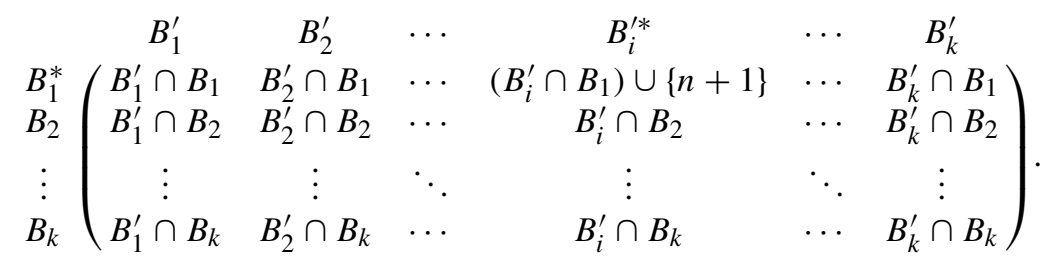

Here, the blocks of $B$ are listed in any order, with empty sets inserted as needed, and the blocks of $B^{\prime}$ are listed in order of least elements, with $k-\# B^{\prime}$ empty sets at the end.

Given $B^{\prime}$, the set of compatible partitions $D_{n, n+1}^{-1}\left(B^{\prime}\right)$ consists of three types depending on the subset $B_{1} \subset[n]$ and the block of $B^{\prime}$ into which $\{n+1\}$ is inserted. Let $B^{\prime \prime} \in D_{n, n+1}^{-1}\left(B^{\prime}\right)$ be the partition of $[n+1]$ obtained by inserting $n+1$ in $B^{\prime}$. Either

(i) $n+1$ is inserted into a block $B_{i}^{\prime}$ such that $B_{i}^{\prime} \cap B_{1} \neq \varnothing$, implying that $\# B_{\mid B_{1}^{*}}^{\prime \prime}=\# B_{\mid B_{1}}^{\prime}$;

(ii) $n+1$ is inserted into a block $B_{i}^{\prime} \neq \varnothing$ such that $B_{i}^{\prime} \cap B_{1}=\varnothing$, implying that $\# B_{\mid B_{1}^{*}}^{\prime \prime}=$ $\# B_{\mid B_{1}}^{\prime}+1$; or

(iii) $n+1$ is inserted into $B^{\prime}$ as a singleton block, implying that $\# B_{\mid B_{1}^{*}}^{\prime \prime}=\# B_{\mid B_{1}}^{\prime}+1$ and $\# B^{\prime \prime}=\# B^{\prime}+1$; we denote this partition by $B_{\varnothing}^{\prime}$. 
There are $k-\# B^{\prime}$ empty columns in which $\{n+1\}$ can be inserted as a singleton in $B^{\prime}$, as in (iii). For $B^{\prime \prime}$ obtained by (ii), the restriction of $B^{\prime \prime}$ to $B_{1}^{*}$ coincides with the restriction of $B_{\varnothing}^{\prime}$ to $B_{1}^{*}$, so each of these restrictions has the same probability under $\varrho_{v}$. For notational convenience in the following calculation, let $D_{1}$ be those elements of $D_{n, n+1}^{-1}\left(B^{\prime}\right)$ which satisfy condition (i) above and $\mathscr{D}_{2}$ those which satisfy condition (ii).

We have

$$
\begin{aligned}
& p_{n+1}\left(B^{*}, D_{n, n+1}^{-1}\left(B^{\prime}\right) ; v\right) \\
& =\sum_{B^{\prime \prime} \in D_{n, n+1}^{-1}\left(B^{\prime}\right)} \frac{k !}{\left(k-\# B^{\prime \prime}\right) !} \prod_{b \in B^{*}} \frac{\left(k-\# B_{\mid b}^{\prime \prime}\right) !}{k !} \varrho_{v}\left(B_{\mid b}^{\prime \prime}\right) \\
& =\frac{k !}{\left(k-\# B^{\prime}\right) !} \prod_{b \in B} \frac{\left(k-\# B_{\mid b}^{\prime}\right) !}{k !} \\
& \times\left[\sum_{B^{\prime \prime} \in D_{1}} \prod_{b \in B^{*}} \varrho_{v}\left(B_{\mid b}^{\prime \prime}\right)\right. \\
& \left.+\sum_{B^{\prime \prime} \in D_{2}} \frac{1}{k-\# B_{\mid B_{1}}^{\prime}} \prod_{b \in B^{*}} \varrho_{v}\left(B_{\mid b}^{\prime \prime}\right)+\frac{k-\# B^{\prime}}{k-\# B_{\mid B_{1}}^{\prime}} \prod_{b \in B^{*}} \varrho_{v}\left(B_{\varnothing \mid b}^{\prime}\right)\right] \\
& =\frac{k !}{\left(k-\# B^{\prime}\right) !} \prod_{b \in B} \frac{\left(k-\# B_{\mid b}^{\prime}\right) !}{k !} \prod_{\left\{b \in B^{*}: b \neq B_{1}^{*}\right\}} \varrho_{v}\left(B_{\mid b}^{\prime}\right) \\
& \times\left[\sum_{B^{\prime \prime} \in D_{1}} \varrho_{\nu}\left(B_{\mid B_{1}^{*}}^{\prime \prime}\right)+\sum_{B^{\prime \prime} \in D_{2}} \frac{1}{k-\# B_{\mid B_{1}}^{\prime}} \varrho_{\nu}\left(B_{\mid B_{1}^{*}}^{\prime \prime}\right)+\frac{k-\# B^{\prime}}{k-\# B_{\mid B_{1}}^{\prime}} \varrho_{\nu}\left(B_{\varnothing \mid B_{1}^{*}}^{\prime}\right)\right] \\
& =\frac{k !}{\left(k-\# B^{\prime}\right) !} \prod_{b \in B} \frac{\left(k-\# B_{\mid b}^{\prime}\right) !}{k !} \prod_{\left\{b \in B: b \neq B_{1}^{*}\right\}} \varrho_{\mathcal{v}}\left(B_{\mid b}^{\prime}\right)\left[\sum_{B^{\prime \prime} \in D_{1}} \varrho_{\nu}\left(B_{\mid B_{1}^{*}}^{\prime \prime}\right)+\varrho_{\nu}\left(B_{\varnothing \mid B_{1}^{*}}^{\prime}\right)\right] \\
& =\frac{k !}{\left(k-\# B^{\prime}\right) !} \prod_{b \in B} \frac{\left(k-\# B_{\mid b}^{\prime}\right) !}{k !} \prod_{\left\{b \in B: b \neq B_{1}^{*}\right\}} \varrho_{v}\left(B_{\mid b}^{\prime}\right)\left[\sum_{B^{\prime \prime} \in D_{\# B_{1}, \# B_{1}+1}^{-1}\left(B_{\mid B_{1}}^{\prime}\right)} \varrho_{v}\left(B^{\prime \prime}\right)\right] \\
& =\frac{k !}{\left(k-\# B^{\prime}\right) !} \prod_{b \in B} \frac{\left(k-\# B_{\mid b}^{\prime}\right) !}{k !} \prod_{\left\{b \in B: b \neq B_{1}^{*}\right\}} \varrho_{v}\left(B_{\mid b}^{\prime}\right)\left[\varrho_{v}\left(B_{\mid B_{1}}^{\prime}\right)\right] \\
& =\frac{k !}{\left(k-\# B^{\prime}\right) !} \prod_{b \in B} \frac{\left(k-\# B_{\mid b}^{\prime}\right) !}{k !} \prod_{b \in B} \varrho_{v}\left(B_{\mid b}^{\prime}\right) \\
& =p_{n}\left(B, B^{\prime} ; v\right) \text {. }
\end{aligned}
$$

Here, (3.3) is obtained from (3.2) by factoring $\left(k ! /\left(k-\# B^{\prime}\right) !\right) \prod_{b \in B}\left(k-\# B_{\mid b}^{\prime}\right) ! / k$ ! out of the sum and using observations (i), (ii), and (iii). In (3.4), we use the fact that, for any $B^{\prime \prime} \in D_{2}$, $B_{\mid B_{1}^{*}}^{\prime \prime}=B_{\varnothing \mid B_{1}^{*}}^{\prime}$, and there are \# $B^{\prime}-\# B_{\mid B_{1}}^{\prime}$ elements in $\mathscr{D}_{2}$ according to (ii). Line (3.5) follows by observing that each $B^{\prime \prime} \in \mathscr{D}_{1}$ corresponds to an element of $D_{\# B_{1}, \# B_{1}+1}^{-1}\left(B_{\mid B_{1}}^{\prime}\right)$ and $B_{\varnothing \mid B_{1}^{*}}^{\prime}$ is the element of $D_{\# B_{1}, \# B_{1}+1}^{-1}\left(B_{\mid B_{1}}^{\prime}\right)$ obtained by inserting $\{n+1\}$ as a singleton in $B_{\mid B_{1}}^{\prime}$. Finally, (3.6) follows from (3.5) by consistency of the paintbox process. This completes the proof. 
The following result is immediate by finite exchangeability, consistency of (3.1) for every $n$, and Kolmogorov's extension theorem (see [6, Theorem 36.1]).

Theorem 3.1. There exists a transition probability $p(\cdot, \cdot ; v)$ on $\left(\mathcal{P}^{(k)}, \sigma\left(\bigcup_{n} \mathcal{P}_{[n]}^{(k)}\right)\right)$ whose finite-dimensional restrictions are given by (3.1).

We call the discrete-time process governed by $p(\cdot, \cdot ; v)$ the $\varrho_{v}$-Markov chain with state space $\mathcal{P}^{(k)}$.

\subsection{Equilibrium measure}

From (3.1), it is clear that, for each $n, k \in \mathbb{N}$ and $B, B^{\prime} \in \mathcal{P}_{[n]}^{(k)}, p_{n}\left(B, B^{\prime} ; v\right)$ is strictly positive provided $v$ is such that $v(s)>0$ for some $s=\left(s_{1}, \ldots, s_{k}\right) \in \mathcal{P}_{\mathrm{m}}^{(k)}$ with $s_{k}>0$. Under this condition, the finite-dimensional chains are aperiodic and irreducible on $\mathcal{P}_{[n]}^{(k)}$, and, therefore, have a unique stationary distribution. In fact, the finite-dimensional chains based on $v$ are aperiodic and irreducible provided $v$ is not degenerate at $(1,0, \ldots, 0) \in \mathcal{P}_{\mathrm{m}}^{(k)}$. The existence of a unique stationary distribution for each $n$ implies that there is a unique stationary probability measure on $\left(\mathcal{P}^{(k)}, \sigma\left(\bigcup_{n} \mathcal{P}_{[n]}^{(k)}\right)\right)$ for $p(\cdot, \cdot ; v)$ from Theorem 3.1.

Proposition 3.3. Let $v$ be a measure on $\mathcal{P}_{\mathrm{m}}^{(k)}$ such that $v$ is nondegenerate at $(1,0, \ldots, 0) \in$ $\mathcal{P}_{\mathrm{m}}^{(k)}$. Then there exists a unique stationary distribution $\theta_{n}(\cdot ; v)$ for $p_{n}(\cdot, \cdot ; v)$ for each $n \geq 1$.

Proof. Fix $n \in \mathbb{N}$, and let $v$ be any measure on $\mathcal{P}_{\mathrm{m}}^{(k)}$ other than that which puts unit mass at $(1,0, \ldots, 0)$. For $B=\left(B_{1}, \ldots, B_{m}\right) \in \mathcal{P}_{[n]}^{(k)},(3.1)$ gives the transition probability

$$
p_{n}(B, B ; v)=\frac{k !}{(k-m) !} \prod_{i=1}^{m} \frac{1}{k} \varrho_{v}\left(B_{i}\right)
$$

and $\varrho_{v}\left(B_{i}\right)=\varrho_{v}\left(\left[\# B_{i}\right]\right)>0$ for each $i=1, \ldots, m$. Hence, $p_{n}(B, B ; \nu)>0$ for every $B \in \mathcal{P}_{[n]}^{(k)}$ and the chain is aperiodic.

To see that the chain is irreducible, let $B, B^{\prime} \in \mathcal{P}_{[n]}^{(k)}$ and let $1_{n}$ denote the one block partition of $[n]$. Then

$$
p_{n}\left(B, 1_{n} ; v\right)=k \prod_{b \in B} \frac{1}{k} \varrho_{\nu}([\# b])>0
$$

and, since $v$ is not degenerate at $(1,0, \ldots, 0)$, there exists a path $1_{n} \mapsto B^{\prime}$ by recursively partitioning $1_{n}$ until it coincides with $B^{\prime}$. For instance, let $B^{\prime}:=\left(B_{1}^{\prime}, \ldots, B_{m}^{\prime}\right) \in \mathcal{P}^{(k)}$. One such path from $1_{n}$ to $B^{\prime}$ is

$$
1_{n} \rightarrow\left(B_{1}^{\prime}, \bigcup_{i=2}^{m} B_{i}^{\prime}\right) \rightarrow\left(B_{1}^{\prime}, B_{2}^{\prime}, \bigcup_{i=3}^{m}\right) \rightarrow \cdots \rightarrow B^{\prime},
$$

which has positive probability for any nondegenerate $v$. Hence, $p_{n}(\cdot, \cdot ; v)$ is irreducible, which establishes the existence of a unique stationary distribution for each $n$.

Theorem 3.2. Let $v$ be a measure on $\mathcal{P}_{\mathrm{m}}^{(k)}$ such that $v((1,0, \ldots, 0))<1$. Then there exists a unique stationary probability measure $\theta(\cdot ; v)$ for the $\varrho_{v}$-Markov chain on $\mathcal{P}^{(k)}$.

Proof. For $v$ satisfying this condition, Proposition 3.3 shows that a stationary distribution exists for each $n \geq 1$. Let $\left(\theta_{n}(\cdot ; \nu), n \geq 1\right)$ be the collection of stationary distributions for the finite-dimensional transition probabilities $\left(p_{n}(\cdot, \cdot ; v), n \geq 1\right)$. We now show that the $\theta_{n}$ are consistent and finitely exchangeable for each $n$. 
Fix $n \in \mathbb{N}$, and let $B \in \mathcal{P}_{[n]}^{(k)}$. Then stationarity of $\theta_{n}(\cdot ; v)$ implies that

$$
\sum_{B^{\prime} \in \mathcal{P}_{[n]}^{(k)}} \theta_{n}\left(B^{\prime} ; v\right) p_{n}\left(B^{\prime}, B ; v\right)=\theta_{n}(B ; v) .
$$

Now write $\theta_{n}(\cdot) \equiv \theta_{n}(\cdot ; \nu)$ and $p_{n}(\cdot, \cdot) \equiv p_{n}(\cdot, \cdot ; \nu)$ for convenience, and let $B^{\prime} \in \mathcal{P}_{[n]}^{(k)}$. We have

$$
\begin{aligned}
\underbrace{\sum_{B^{\prime \prime} \in D_{n, n+1}^{-1}\left(B^{\prime}\right)} \theta_{n+1}\left(B^{\prime \prime}\right)}_{\left(\theta_{n+1} D_{n, n+1}^{-1}\right)\left(B^{\prime}\right)} & =\sum_{B^{\prime \prime} \in D_{n, n+1}^{-1}\left(B^{\prime}\right)} \sum_{B^{*} \in \mathcal{P}_{[n+1]}^{(k)}} \theta_{n+1}\left(B^{*}\right) p_{n+1}\left(B^{*}, B^{\prime \prime}\right) \\
& =\sum_{B^{*} \in \mathcal{P}_{[n+1]}^{(k)}} \theta_{n+1}\left(B^{*}\right)\left[\sum_{B^{\prime \prime} \in D_{n, n+1}^{-1}\left(B^{\prime}\right)} p_{n+1}\left(B^{*}, B^{\prime \prime}\right)\right] \\
& =\sum_{B \in \mathcal{P}_{[n]}^{(k)}} \sum_{B^{*} \in D_{n, n+1}^{-1}(B)} \theta_{n+1}\left(B^{*}\right)\left[p_{n}\left(B, B^{\prime}\right)\right] \\
& =\sum_{B \in \mathcal{P}_{[n]}^{(k)}} p_{n}\left(B, B^{\prime}\right) \sum_{B^{*} \in D_{n, n+1}^{-1}(B)} \theta_{n+1}\left(B^{*}\right) \\
& =\sum_{B \in \mathcal{P}_{[n]}^{(k)}} p_{n}\left(B, B^{\prime}\right)\left(\theta_{n+1} D_{n, n+1}^{-1}\right)(B) .
\end{aligned}
$$

So $\theta_{n+1} D_{n, n+1}^{-1}$ is stationary for $p_{n}$, which implies that $\theta_{n} \equiv \theta_{n+1} D_{n, n+1}^{-1}$ by uniqueness and $\theta_{n}$ is consistent for each $n$.

Let $\sigma$ be a permutation of $[n]$. Then, for any $B, B^{\prime} \in \mathcal{P}_{[n]}^{(k)}, p_{n}\left(\sigma(B), \sigma\left(B^{\prime}\right)\right)=p_{n}\left(B, B^{\prime}\right)$ by exchangeability of $p_{n}$. It follows that $\theta_{n}$ is finitely exchangeable for each $n$ since

$$
\sum_{B \in \mathcal{P}_{[n]}^{(k)}} \theta_{n}(\sigma(B)) p_{n}\left(\sigma(B), \sigma\left(B^{\prime}\right)\right)=\theta_{n}\left(\sigma\left(B^{\prime}\right)\right)
$$

by stationarity, and $p_{n}\left(\sigma(B), \sigma\left(B^{\prime}\right)\right)=p_{n}\left(B, B^{\prime}\right)$ implies that

$$
\sum_{B \in \mathcal{P}_{[n]}^{(k)}} \theta_{n}(\sigma(B)) p_{n}\left(B, B^{\prime}\right)=\theta_{n}\left(\sigma\left(B^{\prime}\right)\right) .
$$

Hence, $\theta_{n} \circ \sigma$ is stationary for $p_{n}$ and $\theta_{n} \equiv \theta_{n} \circ \sigma$ by uniqueness.

Kolmogorov consistency implies that there exists a unique exchangeable stationary probability measure $\theta$ on $\mathcal{P}^{(k)}$ whose restriction to $[n]$ is $\theta_{n}$ for each $n \in \mathbb{N}$. This completes the proof.

\section{The $\varrho_{v}$-Markov process in continuous time}

Let $\lambda>0$, let $v$ be a measure on $\mathcal{P}_{\mathrm{m}}^{(k)}$, and, for each $n \in \mathbb{N}$, define Markovian infinitesimal jump rates for a Markov process on $\mathcal{P}_{[n]}^{(k)}$ by

$$
q_{n}\left(B, B^{\prime} ; v\right)= \begin{cases}\lambda p_{n}\left(B, B^{\prime} ; v\right), & B \neq B^{\prime}, \\ 0, & \text { otherwise, }\end{cases}
$$

where $p_{n}$ is as in (3.1). The infinitesimal generator, $Q_{n}^{v}$, of the process on $\mathcal{P}_{[n]}^{(k)}$ governed by 
$q_{n}$ has entries

$$
Q_{n}^{v}\left(B, B^{\prime}\right)=\lambda \times \begin{cases}p_{n}\left(B, B^{\prime} ; v\right), & B \neq B^{\prime}, \\ p_{n}(B, B ; v)-1, & B=B^{\prime} .\end{cases}
$$

We now construct a Markov process $B:=(B(t), t \geq 0)$ in continuous time whose finitedimensional transition rates are given by (4.1).

Definition 4.1. A process $B:=(B(t), t \geq 0)$ on $\mathcal{P}^{(k)}$ is a $\varrho_{v}$-Markov process if, for each $n \in \mathbb{N}, B_{\mid[n]}$ is a Markov process on $\mathcal{P}_{[n]}^{(k)}$ with $Q$-matrix $Q_{n}^{v}$ as in (4.2).

A process on $\mathcal{P}^{(k)}$ whose finite-dimensional restrictions are governed by $Q_{n}^{v}$ can be constructed according to the matrix construction from Section 3 by permitting only transitions $B \mapsto B^{\prime}$ for $B^{\prime} \neq B$, where $B, B^{\prime} \in \mathcal{P}_{[n]}^{(k)}$, and adding a hold time which is exponentially distributed with mean $-1 / Q_{n}^{v}(B, B)$.

Proposition 4.1. For a measure v on $\mathcal{P}_{\mathrm{m}}^{(k)}$, let $\left(Q_{n}^{v}\right)_{n \in \mathbb{N}}$ be the collection of $Q$-matrices in (4.2). For every $n \in \mathbb{N}$, the entries of $Q_{n}^{v}$ satisfy (2.1).

Proof. Fix $n \in \mathbb{N}$, and let $B, B^{\prime} \in \mathcal{P}_{[n]}^{(k)}$ such that $B \neq B^{\prime}$. Then

$$
Q_{n}^{v}\left(B, B^{\prime}\right)=\sum_{B^{\prime \prime} \in D_{n, n+1}^{-1}\left(B^{\prime}\right)} Q_{n+1}^{v}\left(B_{*}, B^{\prime \prime}\right)
$$

for all $B_{*} \in D_{n, n+1}^{-1}(B)$ by the consistency of $p_{n}$ from Proposition 3.2.

For $B^{\prime}=B$ and $B_{*} \in D_{n, n+1}^{-1}(B)$, we have

$$
\begin{aligned}
\sum_{B^{\prime \prime} \in D_{n, n+1}^{-1}(B)} Q_{n+1}^{v}\left(B_{*}, B^{\prime \prime}\right) & =Q_{n+1}^{v}\left(B_{*}, B_{*}\right)+\sum_{B^{\prime \prime} \in D_{n, n+1}^{-1}(B) \backslash\left\{B_{*}\right\}} Q_{n+1}^{v}\left(B_{*}, B^{\prime \prime}\right) \\
& =\lambda\left[p_{n+1}\left(B_{*}, B_{*} ; v\right)-1+\sum_{B^{\prime \prime} \in D_{n, n+1}^{-1}(B) \backslash\left\{B_{*}\right\}} p_{n+1}\left(B_{*}, B^{\prime \prime} ; v\right)\right] \\
& =\lambda\left[\sum_{B^{\prime \prime} \in D_{n, n+1}^{-1}(B)} p_{n+1}\left(B_{*}, B^{\prime \prime} ; v\right)-1\right] \\
& =\lambda\left(p_{n}(B, B ; v)-1\right) \\
& =Q_{n}^{v}(B, B) .
\end{aligned}
$$

Theorem 4.1. For each measure $v$ on $\mathcal{P}_{\mathrm{m}}^{(k)}$, there exists a Markov process $(B(t), t \geq 0)$ on $\mathcal{P}^{(k)}$ which has finite-dimensional transition rates given in (4.1).

Proof. Let $v$ be a measure on $\mathcal{P}_{\mathrm{m}}^{(k)}$, and let $\left(B_{\mid[n]}(t), t \geq 0\right)_{n \in \mathbb{N}}$ be the collection of restrictions of a $\varrho_{v}$-Markov process with consistent $Q$-matrices $\left(Q_{n}^{v}\right)_{n \in \mathbb{N}}$ as in (4.2). For each $n, Q_{n}^{v}$ is finitely exchangeable and consistent with $Q_{n+1}^{v}$ by Proposition 4.1 , which is sufficient for $B_{\mid[n]}$ to be consistent with $B_{\mid[n+1]}$ for every $n$. Kolmogorov's extension theorem implies that there exist transition rates, $Q^{\nu}$, on $\mathcal{P}^{(k)}$ such that, for every $B, B^{\prime} \in \mathcal{P}_{[n]}^{(k)}$,

$$
Q_{n}^{v}\left(B, B^{\prime}\right)=Q^{v}\left(B_{*},\left\{B^{\prime \prime} \in \mathcal{P}^{(k)}: B_{\mid[n]}^{\prime \prime}=B^{\prime}\right\}\right)
$$

for every $B_{*} \in\left\{B^{\prime \prime} \in \mathcal{P}^{(k)}: B_{\mid[n]}^{\prime \prime}=B\right\}$.

Finally, for every $B \in \mathcal{P}_{[n]}^{(k)}, Q_{n}^{v}\left(B, \mathcal{P}_{[n]}^{(k)} \backslash\{B\}\right)=\lambda\left(1-p_{n}(B, B ; v)\right)<\infty$ so that the sample paths of $B_{\mid[n]}$ are càdlàg for every $n$, which implies that $B$ is càdlàg. 
Corollary 4.1. For $v$ which satisfies the condition of Theorem 3.2, the continuous-time process $B:=(B(t), t \geq 0)$ with finite-dimensional rates $q_{n}(\cdot, \cdot ; v)$ in (4.1) has unique stationary distribution $\theta(\cdot ; v)$ from Theorem 3.2 .

Proof. For each $n \in \mathbb{N}$, let $\theta_{n}(\cdot ; v)$ be the unique finite-dimensional stationary distribution of $p_{n}(\cdot, \cdot ; v)$ from (3.1). It is easy to verify that, for each $n \in \mathbb{N}, \Theta_{n}^{v}:=\left(\theta_{n}(B ; v), B \in \mathcal{P}_{[n]}^{(k)}\right)$ satisfies

$$
\left(\Theta_{n}^{v}\right)^{t} Q_{n}^{v}=0,
$$

which establishes that $\Theta_{n}^{v}$ is stationary for $Q_{n}^{v}$ for every $n$. The rest of the proof follows by Theorem 3.2.

\subsection{Poissonian construction}

From the matrix construction at the beginning of Section 3, a consistent family of finitedimensional Markov processes with transition rates as in (4.1) can be constructed by a Poisson point process on $\mathbb{R}^{+} \times \prod_{i=1}^{k} \mathcal{P}^{(k)}$ as follows. Let

$$
P=\left\{\left(t, C_{1}, \ldots, C_{k}\right)\right\} \subset \mathbb{R}^{+} \times \prod_{i=1}^{k} \mathcal{P}^{(k)}
$$

be a Poisson point process with intensity measure $\mathrm{d} t \otimes \lambda \varrho_{\nu}^{(k)}$ for some measure $v$ on $\mathcal{P}_{\mathrm{m}}^{(k)}$ and $\lambda>0$, where $\varrho_{v}^{(k)}$ is the product measure $\varrho_{v} \otimes \cdots \otimes \varrho_{v}$ on $\prod_{i=1}^{k} \mathcal{P}^{(k)}$.

Construct an exchangeable process $B:=(B(t), t \geq 0)$ on $\mathscr{P}^{(k)}$ by taking $\pi \in \mathcal{P}^{(k)}$ to be some exchangeable random partition and setting $B(0)=\pi$.

For each $n \in \mathbb{N}$, set $B_{\mid[n]}(0)=\pi_{\mid[n]}$ and

- if $t$ is not an atom time for $P$ then $B_{\mid[n]}(t)=B_{\mid[n]}(t-)$;

- if $t$ is an atom time for $P$ so that $\left(t, C_{1}, \ldots, C_{k}\right) \in P$ then, independently of $(B(s)$, $s<t)$ and $\left(t, C_{1}, \ldots, C_{k}\right)$, generate $\sigma_{1}, \ldots, \sigma_{k}$ i.i.d. uniform random permutations of $[k]$ and construct $B^{\prime}$ from the set partition induced by the column totals $\left(C_{.1}, \ldots, C_{. k}\right)$ of

$$
\begin{array}{cccc}
C_{.1} & C_{.2} & \cdots & C_{. k} \\
B_{1} \\
B_{2} \\
\vdots \\
B_{k}
\end{array}\left(\begin{array}{cccc}
C_{1, \sigma_{1}(1)} \cap B_{1} & C_{1, \sigma_{1}(2)} \cap B_{1} & \cdots & C_{1, \sigma_{1}(k)} \cap B_{1} \\
C_{2, \sigma_{2}(1)} \cap B_{2} & C_{2, \sigma_{2}(2)} \cap B_{2} & \ldots & C_{2, \sigma_{2}(k)} \cap B_{2} \\
\vdots & \vdots & \ddots & \vdots \\
C_{k, \sigma_{k}(1)} \cap B_{k} & C_{k, \sigma_{k}(2)} \cap B_{k} & \ldots & C_{k, \sigma_{k}(k)} \cap B_{k}
\end{array}\right)=: B \cap C^{\sigma},
$$

where $\left(B_{1}, \ldots, B_{k}\right)$ are the blocks of $B=B_{\mid[n]}(t-)$ listed in order of their least element, with $k-\# B$ empty sets at the end of the list.

- If $B^{\prime} \neq B$ then $B_{\mid[n]}(t)=B^{\prime}$.

- If $B^{\prime}=B$ then $B_{\mid[n]}(t)=B_{\mid[n]}(t-)$.

Proposition 4.2. The above process $B$ is a Markov process on $\mathcal{P}^{(k)}$ with transition matrix $Q^{v}$ defined by Theorem 4.1.

Proof. This is clear from the consistency of both the paintbox process $\varrho_{v}$ and the $Q_{n}^{v}$-matrices for every $n$ and the fact that, by this construction, for any $n$ such that $B_{\mid[n]}(t)=\pi$, then $B_{[n] \mid[m]}(t)=D_{m, n}(\pi)$ for all $m<n$ and $B_{\mid[p]}(t) \in D_{n, p}^{-1}(\pi)$ for all $p>n$. 
Let $\mathrm{P}_{t}$ be the semigroup of a $\varrho_{\nu}$-Markov process $B(\cdot)$, i.e. for any continuous $\varphi: \mathcal{P}^{(k)} \rightarrow \mathbb{R}$,

$$
\mathrm{P}_{t} \varphi(\pi):=\mathrm{E}_{\pi} \varphi(B(t)),
$$

the expectation of $\varphi(B(t))$ given $B(0)=\pi$.

Corollary 4.2. $A \varrho_{v}$-Markov process has the Feller property, i.e.

- for each continuous function $\varphi: \mathcal{P}^{(k)} \rightarrow \mathbb{R}$ and each $\pi \in \mathcal{P}$, we have

$$
\lim _{t \downarrow 0} \mathrm{P}_{t} \varphi(\pi)=\varphi(\pi) ;
$$

- for all $t>0, \pi \mapsto \mathrm{P}_{t} \varphi(\pi)$ is continuous.

Proof. The proof follows the same program as the proof of [2, Corollary 6].

Let

$$
C_{f}:=\left\{f: \mathcal{P}^{(k)} \rightarrow \mathbb{R}: \text { there exists } n \in \mathbb{N} \text { such that } \pi_{\mid[n]}=\pi_{\mid[n]}^{\prime} \Rightarrow f(\pi)=f\left(\pi^{\prime}\right)\right\}
$$

be a set of functions which is dense in the space of continuous functions from $\mathcal{P}^{(k)} \rightarrow \mathbb{R}$. It is clear that, for $g \in C_{f}, \lim _{t \downarrow 0} \mathrm{P}_{t} g(\pi)=g(\pi)$ since the first jump time of $B(\cdot)$ is an exponential variable with finite mean. The first point follows for all continuous functions $\mathcal{P}^{(k)} \rightarrow \mathbb{R}$ by the denseness of $C_{f}$.

For the second point, let $\pi, \pi^{\prime} \in \mathcal{P}^{(k)}$ be such that $d\left(\pi, \pi^{\prime}\right)<1 / n$ and use the same Poisson point process $P$ to construct two $\varrho_{\nu}$-Markov processes, $B(\cdot)$ and $B^{\prime}(\cdot)$, with starting points $\pi$ and $\pi^{\prime}$, respectively. By the construction, $B_{\mid[n]}=B_{\mid[n]}^{\prime}$ and $d\left(B(t), B^{\prime}(t)\right)<1 / n$ for all $t \geq 0$. It follows that, for any continuous $g, \pi \mapsto \mathrm{P}_{t} g(\pi)$ is continuous.

This allows us to characterize the $\varrho_{\nu}$-Markov process in terms of its infinitesimal generator. Let $B:=(B(t), t \geq 0)$ be the $\varrho_{v}$-Markov process on $\mathcal{P}^{(k)}$ with transition rates characterized by $\left(q_{n}\right)_{n \in \mathbb{N}}$ as in (4.1). The infinitesimal generator, $\mathcal{A}$, of $B$ is given by

$$
\mathcal{A}(f)(\pi)=\int_{\mathcal{P}^{(k)}}\left(f\left(\pi^{\prime}\right)-f(\pi)\right) Q^{v}\left(\pi, \mathrm{d} \pi^{\prime}\right)
$$

for every $f \in C_{f}$.

\section{Asymptotic frequencies}

Definition 5.1. A subset $A \subset \mathbb{N}$ is said to have asymptotic frequency $\lambda$ if

$$
\lambda:=\lim _{n \rightarrow \infty} \frac{\#\{i \leq n: i \in A\}}{n}
$$

exists, and a random partition $B:=\left(B_{1}, B_{2}, \ldots\right) \in \mathcal{P}$ is said to have asymptotic frequencies if each block of $B$ has an asymptotic frequency almost surely.

Adopting the notation of [2], let $\Lambda(B)=\left(\left\|B_{1}\right\|,\left\|B_{2}\right\|, \ldots\right)^{\downarrow}$ be the decreasing arrangement of asymptotic frequencies of a partition $B=\left(B_{1}, B_{2}, \ldots\right) \in \mathcal{P}$ which possesses asymptotic frequencies, some of which could be 0 .

According to Kingman's representation theorem (see Theorem 2.2 of [17]), any exchangeable random partition of $\mathbb{N}$ possesses asymptotic frequencies. Intuitively, this is a consequence of generating an exchangeable random partition of $\mathbb{N}$ by the paintbox process. 
The process described in Section 3 assigns only positive probability to transitions involving two partitions with at most $k$ blocks. From the Poissonian construction of the transition rates in Section 4.1, it is evident that the states of $B=(B(t), t \geq 0)$ will have at most $k$ blocks almost surely. Moreover, the description of the transition rates in terms of the paintbox process allows us to describe the associated measure-valued process of $B:=(B(t), t \geq 0)$ characterized by $\lambda$ and $\nu$.

\subsection{Poissonian construction}

Consider the following Poissonian construction of a measure-valued process $X:=(X(t)$, $t \geq 0)$ on $\mathcal{P}_{\mathrm{m}}^{(k)}$. For any $k \in \mathbb{N}, \lambda>0$, and $v$ as above, let $P^{\prime}=\left\{\left(t, P_{1}^{\prime}, \ldots, P_{k}^{\prime}\right)\right\} \subset$ $\mathbb{R}^{+} \times \prod_{i=1}^{k} \mathcal{P}_{\mathrm{m}}^{(k)}$ be a Poisson point process with intensity measure $\mathrm{d} t \otimes \lambda v^{(k)}$, where $v^{(k)}$ is the product measure $v \otimes \ldots \otimes v$ on $\prod_{i=1}^{k} \mathcal{P}_{\mathrm{m}}^{(k)}$.

Construct a process $X:=(X(t), t \geq 0)$ on $\mathcal{P}_{\mathrm{m}}^{(k)}$ by generating $p_{0}$ from some probability distribution on $\mathcal{P}_{\mathrm{m}}^{(k)}$. Set $X(0)=p_{0}$ and

- if $t$ is not an atom time for $P^{\prime}$ then $X(t)=X(t-)$;

- if $t$ is an atom time for $P^{\prime}$ so that $\left(t, P_{1}^{\prime}, \ldots, P_{k}^{\prime}\right) \in P^{\prime}$, with $P_{j}^{\prime}=\left(P_{1}^{j}, \ldots, P_{k}^{j}\right)$ for each $j=1, \ldots, k$, and $X(t-)=\left(x_{1}, \ldots, x_{k}\right) \in \mathcal{P}_{\mathrm{m}}^{(k)}$, then, independently of $(X(s)$, $s<t)$ and $\left(t, P_{1}^{\prime}, \ldots, P_{k}^{\prime}\right)$, generate $\sigma_{1}, \ldots, \sigma_{k}$ i.i.d. uniform random permutations of $[k]$ and construct $X(t)$ from the marginal column totals of

$$
\begin{array}{cccc}
P_{1} & P_{2} & \cdots & P_{k} \\
x_{1} \\
x_{2} \\
\vdots \\
x_{k}
\end{array}\left(\begin{array}{cccc}
x_{1} P_{\sigma_{1}(1)}^{1} & x_{1} P_{\sigma_{1}(2)}^{1} & \cdots & x_{1} P_{\sigma_{1}(k)}^{1} \\
x_{2} P_{\sigma_{2}(1)}^{2} & x_{2} P_{\sigma_{2}(2)}^{2} & \cdots & x_{2} P_{\sigma_{2}(k)}^{2} \\
\vdots & \vdots & \ddots & \vdots \\
x_{k} P_{\sigma_{k}(1)}^{k} & x_{k} P_{\sigma_{k}(2)}^{k} & \cdots & x_{k} P_{\sigma_{k}(k)}^{k}
\end{array}\right) \text {, }
$$

i.e. set $X(t)=\left(P_{1}, P_{2}, \ldots, P_{k}^{\cdot}\right)^{\downarrow}:=\left(\sum_{i=1}^{k} x_{i} P_{\sigma_{i}(j)}^{i}, 1 \leq j \leq k\right)^{\downarrow}$.

Theorem 5.1. Let $X:=(X(t), t \geq 0)$ be the process constructed above. Then $X \stackrel{\mathrm{D}}{=} \Lambda(B)$, where $B:=(B(t), t \geq 0)$ is the $\varrho_{\nu}$-Markov process from Theorem 4.1, and ' $=$ ' denotes equality in distribution.

Proof. Fix $k \in \mathbb{N}$, and let $v(\cdot)$ be a measure on $\mathcal{P}_{\mathrm{m}}^{(k)}$.

In the description of the sample paths of $B$ in Section 4 , note that generating $\left(C_{1}, \ldots, C_{k}\right) \sim$ $\varrho_{v}^{(k)}$ is equivalent to first generating $s_{i} \sim v$ independently for each $i=1, \ldots, k$, and then generating random partitions $C_{i}$ by sampling from $s_{i}$ for each $i=1, \ldots, k$. Finally, $B_{i}^{\prime}$ is set equal to the marginal total of column $i$ of the matrix $B \cap C^{\sigma}$, where $\sigma:=\left(\sigma_{1}, \ldots, \sigma_{k}\right)$ is an i.i.d. collection of uniform random permutations of $[k]$. Hence, we can couple the two processes $X$ and $B$ together using the Poisson point process $P^{\prime}$ described above.

Let $X$ evolve according to the Poisson point process $P^{\prime}$ on $\mathbb{R}^{+} \times \prod_{i=1}^{k} \mathcal{P}_{\mathrm{m}}^{(k)}$ as described above. Let $B$ evolve by the modification that if $t$ is an atom time of $P^{\prime}$ then we obtain partitions $\left(C_{1}, \ldots, C_{k}\right)$ by sampling $X^{i}:=\left(X_{1}^{i}, X_{2}^{i}, \ldots\right)$ i.i.d. from $P_{i}^{\prime}$ for each $i=1, \ldots, k$, i.e.

$$
\mathrm{P}\left(X_{1}^{i}=j \mid P_{i}^{\prime}\right)=P_{j}^{i},
$$

and defining the blocks of $C_{i}$ as the equivalence classes of $X^{i}$. Constructed in this way, $\left\|C_{i j}\right\|=P_{j}^{i}$ almost surely for each $i, j=1, \ldots, k$ and $\left(C_{1}, \ldots, C_{k}\right) \sim \varrho_{v}^{(k)}$. 
After obtaining the $C_{i}$, generate, independently of $B, C_{1}, \ldots, C_{k}, P^{\prime}$, i.i.d. uniform permutations $\sigma_{1}, \ldots, \sigma_{k}$ of $[k]$ and proceed as in the construction of Section 4.1 where $B, C_{1}, \ldots, C_{k}$ are arranged in the matrix $B \cap C^{\sigma}$ and the blocks of $B^{\prime}$ are obtained as the marginal column totals of $B \cap C^{\sigma}$. The $(i, j)$ th entry of $B \cap C^{\sigma}$ is $C_{i, \sigma_{i}(j)} \cap B_{i}$ for which we have $\left\|C_{i, \sigma_{i}(j)} \cap B_{i}\right\|=$ $\left\|C_{i, \sigma_{i}(j)}\right\|\left\|B_{i}\right\|=x_{i} P_{\sigma_{i}(j)}^{i}$ almost surely.

By this construction, $B(t)$ is constructed according to a Poisson point process with the same law as that described in Section 4.1, and $B(t)$ possesses ranked asymptotic frequencies which correspond to $X(t)$ almost surely for all $t \geq 0$.

Corollary 5.1. $X(t):=(\Lambda(B(t)), t \geq 0)$ exists almost surely.

\subsection{Equilibrium measure}

Just as the process $(B(t), t \geq 0)$ on $\mathcal{P}^{(k)}$ converges to a stationary distribution, so does its associated measure-valued process $(X(t), t \geq 0)$ from Section 5.1.

Theorem 5.2. The associated measure-valued process $X$ for a $\varrho_{\nu}$-Markov process with unique stationary measure $\theta(\cdot ; v)$ has equilibrium measure $\tilde{\theta}(\cdot ; v)$, the distribution of the ranked frequencies of a $\theta(\cdot ; v)$-partition.

Proof. Proposition 1.4 of [5] states that if a sequence of exchangeable random partitions converges in law on $\mathcal{P}$ to $\pi_{\infty}$ then its sequence of ranked asymptotic frequencies converges in law to $\left|\pi_{\infty}\right|^{\downarrow}$. Hence, from Corollary 4.1 we find that $X$ has equilibrium distribution given by the ranked asymptotic frequencies of a $\theta(\cdot ; v)$-partition.

\section{The $(\alpha, k)$-Markov process}

Pitman [17] discussed a two-parameter family of infinitely exchangeable random partitions called the $(\alpha, \theta)$-process which has finite-dimensional distributions

$$
p_{n}(B ; \alpha, \theta):=\frac{(\theta / \alpha)^{\uparrow \# B}}{\theta^{\uparrow n}} \prod_{b \in B}-(-\alpha)^{\uparrow \# b}
$$

for $(\alpha, \theta)$ satisfying either

- $\alpha=-\kappa<0$ and $\theta=m \kappa$ for some $m=1,2, \ldots$; or

- $0 \leq \alpha \leq 1$ and $\theta>-\alpha$.

For $k \in \mathbb{N}$ and $\alpha>0$, a $(-\alpha, k \alpha)$-partition has finite-dimensional distributions

$$
\rho_{n}(B ; \alpha, k)=\frac{k !}{(k-\# B) !} \frac{\prod_{b \in B} \Gamma(\alpha+\# b) / \Gamma(\alpha)}{\Gamma(k \alpha+n) / \Gamma(k \alpha)}
$$

whose support is $\mathcal{P}_{[n]}^{(k)}$.

The distribution of the ranked asymptotic frequencies of an $(\alpha, \theta)$-partition is called the Poisson-Dirichlet distribution with parameter $(\alpha, \theta)$, written $\operatorname{PD}(\alpha, \theta)$.

For notational convenience, introduce the $\alpha$-permanent [14] of an $n \times n$ matrix $K$,

$$
\operatorname{per}_{\alpha} K=\sum_{\sigma \in \jmath_{n}} \alpha^{\# \sigma} \prod_{i=1}^{n} K_{i, \sigma(i)}
$$

where $\# \sigma$ is the number of cycles of the permutation $\sigma$, and note that, when $B \in \mathcal{P}_{[n]}$ is regarded 
as a matrix,

$$
\operatorname{per}_{\alpha} B=\prod_{b \in B} \operatorname{per}_{\alpha} B_{\mid b}=\prod_{b \in B} \frac{\Gamma(\alpha+\# b)}{\Gamma(\alpha)},
$$

which allows us to write (6.1) as

$$
\rho_{n}(B ; \alpha, k)=\frac{k !}{(k-\# B) !} \frac{\operatorname{per}_{\alpha} B}{(k \alpha)^{\uparrow n}},
$$

where $(\beta)^{\uparrow n}=\beta(\beta+1) \cdots(\beta+n-1)$.

We now consider a specific subfamily of reversible $\varrho_{\nu}$-Markov processes for which the transition probabilities can be written down explicitly. For $k \in \mathbb{N}$ and $\alpha>0$, let $v$ be the $\mathrm{PD}(-\alpha / k, \alpha)$ distribution on $\mathcal{P}_{\mathrm{m}}^{(k)}$ and define transition probabilities according to the matrix construction based on $v$ as in Section 3. We call this process the $(\alpha, k)$-Markov process.

Proposition 6.1. The $(\alpha, k)$-Markov process has finite-dimensional transition probabilities

$$
\begin{aligned}
p_{n}\left(B, B^{\prime} ; \alpha, k\right) & =\frac{k !}{\left(k-\# B^{\prime}\right) !} \prod_{b \in B} \frac{\prod_{b^{\prime} \in B^{\prime}} \Gamma\left(\alpha / k+\#\left(b \cap b^{\prime}\right)\right) / \Gamma(\alpha / k)}{\Gamma(\alpha+\# b) / \Gamma(\alpha)} \\
& =\frac{k !}{\left(k-\# B^{\prime}\right) !} \frac{\operatorname{per}_{\alpha / k}\left(B \wedge B^{\prime}\right)}{\operatorname{per}_{\alpha} B}
\end{aligned}
$$

Proof. Theorem 3.2 and Definition 3.3 of [17] shows that the distribution of $B \sim \varrho_{v}$, where $v=\mathrm{PD}(-\alpha / k, \alpha)$, is

$$
\rho_{n}\left(B ; \frac{\alpha}{k}, k\right)=\frac{k !}{(k-\# B) !} \frac{\operatorname{per}_{\alpha / k} B}{(\alpha)^{\uparrow n}} .
$$

Combining this and (3.1) yields (6.3); (6.4) follows from (6.2).

Proposition 6.2. For each $(\alpha, k) \in \mathbb{R}^{+} \times \mathbb{N}$ and $n \in \mathbb{N}, p_{n}(\cdot, \cdot ; \alpha, k)$ defined in Proposition 6.1 is reversible with respect to (6.1) with parameter $(\alpha, k)$.

Proof. Let $\rho_{n}(\cdot ; \alpha, k)$ be the distribution with parameter $(\alpha, k)$ defined in (6.1), and let $p_{n}(\cdot, \cdot ; \alpha, k)$ be as defined in (6.3). For any $B, B^{\prime} \in \mathcal{P}_{[n]}^{(k)}$, it is immediate that

$$
\rho_{n}(B ; \alpha, k) p_{n}\left(B, B^{\prime} ; \alpha, k\right)=\rho_{n}\left(B^{\prime} ; \alpha, k\right) p_{n}\left(B^{\prime}, B ; \alpha, k\right),
$$

which establishes reversibility.

Bertoin [4] discussed some reversible EFC processes which have $\operatorname{PD}(\alpha, \theta)$ distribution as their equilibrium measure, for $0<\alpha<1$ and $\theta>-\alpha$. Here we have shown reversibility with respect to $\operatorname{PD}(\alpha, \theta)$ for $\alpha<0$ and $\theta=-m \alpha$ for $m \in \mathbb{N}$.

The construction of the continuous-time process is a special case of the procedure in Section 4. The measure-valued process $(X(t), t \geq 0)$ based on the $(\alpha, k)$-Markov process has unique stationary measure $\mathrm{PD}(-\alpha, k \alpha)$, the distribution of the ranked frequencies of a partition with finite-dimensional distributions as in (6.1) with parameter $(\alpha, k)$.

\section{Discussion}

The paths of the $\varrho_{\nu}$-Markov process discussed above are confined to $\mathcal{P}^{(k)}$. Unlike the EFC process [2], which has a natural interpretation as a model in certain physical sciences, the 
$\varrho_{\nu}$-Markov process has no clear interpretation as a physical model. However, the matrix construction introduced in Section 3 leads to transition rates which admit a closed-form expression in the case of the $(\alpha, k)$-Markov process.

The $(\alpha, k)$ class of models could be useful as a statistical model for relationships among statistical units which are known to fall into one of $k$ classes. In statistical work, it is important that any observation has positive probability under the specified model. The $(\alpha, k)$-process assigns positive probability to all possible transitions and so any observed sequence of partitions in $\mathcal{P}_{[n]}^{(k)}$ will have positive probability for any choice of $\alpha>0$. In addition, the model is exchangeable, consistent, and reversible, particularly attractive mathematical properties which could have a natural interpretation in certain applications. Future work is intended to explore applications for this model, as well as develop some of the tools necessary for its use in statistical inference.

\section{Acknowledgements}

This work was supported by the National Science Foundation, grant number DMS-0906592. I also thank Peter McCullagh for his many helpful comments throughout the editing of this paper.

\section{References}

[1] Aldous, D. J. (1999). Deterministic and stochastic models for coalescence (aggregation and coagulation): a review of the mean-field theory for probabilists. Bernoulli 5, 3-48.

[2] BerestyCKI, J. (2004). Exchangeable fragmentation-coalescence processes and their equilibrium measures. Electron. J. Prob. 9, 770-824.

[3] Bertoin, J. (2006). Random Fragmentation and Coagulation Processes. Cambridge University Press.

[4] Bertoin, J. (2008). Two-parameter Poisson-Dirichlet measures and reversible exchangeable fragmentationcoagulation processes. Combinatorics Prob. Comput. 17, 329-337.

[5] Bertoin, J. (2010). Exchangeable Coalescents (Lecture Notes for PIMS Summer School in Probability). University of Washington and Microsoft Research.

[6] Billingsley, P. (1995). Probability and Measure, 3rd edn. John Wiley, New York.

[7] Blei, D., Ng, A. And Jordan, M. (2003). Latent Dirichlet allocation. J. Machine Learning Res. 3, 993-1022.

[8] Burke, C. J. and Rosenblatt, M. (1958). A Markovian function of a Markov chain. Ann. Math. Statist. 29, $1112-1122$.

[9] EFron, B. AND Thisted, R. (1976). Estimating the number of unseen species: how many words did Shakespeare know? Biometrika 63, 435-447.

[10] Ewens, W. J. (1972). The sampling theory of selectively neutral alleles. Theoret. Pop. Biol. 3, 87-112.

[11] Fisher, R. A., Corbet, A. S. And Williams, C. B. (1943). The relation between the number of species and the number of individuals in a random sample of an animal population. J. Animal Ecology 12, 42-58.

[12] Kingman, J. F. C. (1978). Random partitions in population genetics. Proc. R. Soc. London A 361, 1-20.

[13] McCullagh, P. (2010). Random permutations and partition models. In International Encyclopedia of Statistical Science, ed. M. Lovric, Springer, Berlin, pp. 1170-1177.

[14] McCullagh, P. and Møller, J. (2005). The permanent process. Adv. Appl. Prob. 38, 873-888.

[15] McCullagh, P. and Yang, J. (2008). How many clusters? Bayesian Anal. 3, 101-120.

[16] McCullagh, P., Pitman, J. And Winkel, M. (2008). Gibbs fragmentation trees. Bernoulli 14, 988-1002.

[17] Pitman, J. (2006). Combinatorial Stochastic Processes (Lecture Notes Math. 1875). Springer, New York. 